\title{
Limited Asymmetry Dependence of Correlations from Single Nucleon Transfer
}

\author{
F. Flavigny, ${ }^{1,2}$ A. Gillibert, ${ }^{1}$ L. Nalpas, ${ }^{1}$ A. Obertelli, ${ }^{1}$ N. Keeley,${ }^{3}$ C. Barbieri ${ }^{4}$ D. Beaumel,${ }^{5}$ S. Boissinot, ${ }^{1}$ G. Burgunder, ${ }^{6}$
} A. Cipollone, ${ }^{4,7,8}$ A. Corsi, ${ }^{1}$ J. Gibelin, ${ }^{9}$ S. Giron, ${ }^{5}$ J. Guillot, ${ }^{5}$ F. Hammache, ${ }^{5}$ V. Lapoux, ${ }^{1}$ A. Matta, ${ }^{5}$ E. C. Pollacco, ${ }^{1}$ R. Raabe, ${ }^{6,2}$ M. Rejmund, ${ }^{6}$ N. de Séreville, ${ }^{5}$ A. Shrivastava, ${ }^{6}$ A. Signoracci, ${ }^{1}$ and Y. Utsuno ${ }^{10}$

${ }^{1}$ CEA, Centre de Saclay, IRFU/Service de Physique Nucléaire, F-91191 Gif-sur-Yvette, France

${ }^{2}$ Instituut voor Kern- en Stralingsfysica, KU Leuven, B-3001 Leuven, Belgium

${ }^{3}$ National Centre for Nuclear Research, ul Andrezja Soltana 7, PL-05400 Otwock, Poland

${ }^{4}$ Department of Physics, University of Surrey, Guilford GU2 7XH, United Kingdom

${ }^{5}$ Institut de Physique Nucléaire Orsay, IN2P3-CNRS, 91406 Orsay Cedex, France

${ }^{6}$ GANIL, BP 55076,14076 Caen Cedex 5, France

${ }^{7}$ Dipartimento di Fisica, Università Sapienza, I-00185 Roma, Italy

${ }^{8}$ INFN, Sezione di Roma, Piazzale Aldo Moro 2, I-00185 Roma, Italy

${ }^{9}$ LPC Caen, ENSICAEN, Université de Caen, CNRS/IN2P3, Caen, France

${ }^{10}$ Advanced Science Research Center, Japan Atomic Energy Agency, Tokai, Ibaraki 319-1195, Japan

(Received 1 August 2012; revised manuscript received 9 January 2013; published 21 March 2013)

Single nucleon pickup reactions were performed with a $18.1 \mathrm{MeV} /$ nucleon ${ }^{14} \mathrm{O}$ beam on a deuterium target. Within the coupled reaction channel framework, the measured cross sections were compared to theoretical predictions and analyzed using both phenomenological and microscopic overlap functions. The missing strength due to correlations does not show significant dependence on the nucleon separation energy asymmetry over a wide range of $37 \mathrm{MeV}$, in contrast with nucleon removal data analyzed within the sudden-eikonal formalism.

DOI: 10.1103/PhysRevLett.110.122503

PACS numbers: $24.50 .+\mathrm{g}$

The existence of single-particle-like modes in nuclei, near the Fermi surface, is particularly important because these are at the basis of the nuclear shell model and thus govern the low energy nuclear dynamics. Yet, they result from nontrivial many-body correlations, which affect energy ordering and filling of active orbits. Spectroscopic factors (SFs) are a unique tool to address the question of correlations as they are strictly linked to the notion of shell occupancies and can be probed using direct reaction cross section measurements [1,2]. Information for stable nuclei was formerly provided by the electromagnetic probe $\left(e, e^{\prime} p\right)$ [3-5]. Even for closed shell nuclei like ${ }^{16} \mathrm{O}$ or ${ }^{208} \mathrm{~Pb}$, a cross section reduction by $30 \%-40 \%$ relative to an independent-particle-based model was observed. Different origins are now well established, like short range correlations [1] and couplings to collective modes at high excitation energy [6] or to the continuum [7]. Single nucleon pickup reactions were also used for stable nuclei yielding results consistent with $\left(e, e^{\prime} p\right)$ measurements $[8,9]$.

For nuclei away from the valley of stability, new approaches have been developed in inverse kinematics at various incident energies, knockout and transfer reactions. From knockout reactions at intermediate energy, a reduction factor $R_{s}$ was deduced as the ratio between the experimental cross section and a theoretical value obtained in a sudden-eikonal approach [10]. A strong dependence was claimed for $R_{S}$ versus the asymmetry (difference in separation energy) $\Delta S=\epsilon\left(S_{p}-S_{n}\right)$ with $\epsilon=+1(-1)$ for proton (neutron) removal reactions, with a reduction as high as $70 \%$ for large positive $\Delta S$ values. This reduction is still not understood and was first accounted for by possible missing correlations in shell-model calculations [10]. Different conclusions were drawn from (i) the possibility of dissipative processes beyond the sudden approximation $[11,12]$, and (ii) transfer reactions at lower incident energies showing no $\Delta S$ dependence of $R_{s}$ [13]. From a theoretical point of view, ab initio calculations suggest only a mild dependence of SFs on $\Delta S[7,14]$, with equal SFs found for the nucleon removals from ${ }^{56} \mathrm{Ni}[6]$ despite significant $\Delta S$ values $( \pm 9.5 \mathrm{MeV})$. Coupled-cluster calculations [7] pointed out a further decrease of proton SFs for isotopes at the neutron dripline, due to coupling to the continuum. This has the substantial effect of enhancing the dependence on $\Delta S$ but still with a much reduced amplitude compared to Ref. [10].

In this Letter, we report on the asymmetry dependence of $R_{s}$ along the oxygen isotopic chain with the study of single nucleon pickup reactions, especially for ${ }^{14} \mathrm{O}$ with large $\Delta S$ values ( $\pm 18.5 \mathrm{MeV}$ for transfer to the gs).

$\mathrm{A}^{14} \mathrm{O}^{8+}$ beam was accelerated at $18.1 \mathrm{MeV} /$ nucleon at the SPIRAL facility (GANIL, Caen) with a mean intensity of $6 \times 10^{4} \mathrm{pps}$. We used deuterated polypropylene $\mathrm{CD}_{2}$ targets: $0.5 \mathrm{mg} / \mathrm{cm}^{2}$ for the $\left(d,{ }^{3} \mathrm{He}\right)$ channel, $1.5 \mathrm{mg} / \mathrm{cm}^{2}$ for the elastic scattering and $8.5 \mathrm{mg} / \mathrm{cm}^{2}$ for the $(d, t)$ channel. The MUST2 array [15] was dedicated to the detection and identification of recoil nuclei: four telescopes at forward angles for ${ }^{3} \mathrm{H}$ and ${ }^{3} \mathrm{He}$ and two telescopes around $90^{\circ}$ relative to the beam axis for elastic scattering. For an exclusive discrimination, the ejectiles were identified in mass and charge in the focal plane of the VAMOS 
magnetic spectrometer [16]. With two beam tracking detectors [17] upstream of the target, the overall angular resolution was improved down to an average value of $0.2^{\circ}$ for a distance of $18 \mathrm{~cm}$ between the target and the forward telescopes. The resolution in excitation energy was found to be $720 \mathrm{keV}$ (FWHM) for elastic scattering. Besides the statistical uncertainty, the experimental cross sections were extracted with the following uncertainties: (i) target thickness (4\%), (ii) beam intensity (3\%), (iii) efficiencies $(5 \%)$, and (iv) background suppression for the $\left(d,{ }^{3} \mathrm{He}\right)$ channel (5\%).

${ }^{14} \mathrm{O}(d, d)$ elastic scattering at $17.8 \mathrm{MeV} /$ nucleon (midtarget) is shown in Fig. 1. The angular distributions for single nucleon pickup are shown in Fig. 2. For the $\left(d,{ }^{3} \mathrm{He}\right)$ channel, two peaks are observed with similar intensities [Fig. 2(b)]. They correspond to the ground state and 3/2 resonance at $3.5 \mathrm{MeV}$ in ${ }^{13} \mathrm{~N}$, the latter being unbound to proton emission [18] and observed with the MUST2 array alone. The setting of the MUST2 array was optimized for the detection of ${ }^{3} \mathrm{H}$ rather than ${ }^{3} \mathrm{He}$, which explains the limited angular coverage. In addition to our results, we included published differential cross sections for reactions at about the same incident energy on ${ }^{16} \mathrm{O}$ and ${ }^{18} \mathrm{O}$ targets in direct kinematics [19-21]. Differential cross sections were calculated with the FRESCO code [22], using the coupled reaction channel (CRC) and coupled discretized continuum channel (CDCC) approaches to include couplings to the single nucleon pickup reactions and to the deuteron breakup, respectively. The entrance channel deuteron potential was obtained by Watanabe-type folding of the central part of a global nucleon-nucleus optical potential over the deuteron internal wave function. The transfer steps included full complex remnant terms and nonorthogonality corrections. The exit channel ${ }^{3} \mathrm{H}$ and ${ }^{3} \mathrm{He}$ optical model potentials were obtained from the systematics of Refs. [23,24]. A set of four nucleon-nucleus potentials [25-28] in the entrance channel, together with the two exit channel potentials $[23,24]$ were used to estimate the uncertainty introduced by the choice of potentials. In Figs. 1 and 2, the results of the calculation using the potentials of Refs. [24,25] in the entrance and exit

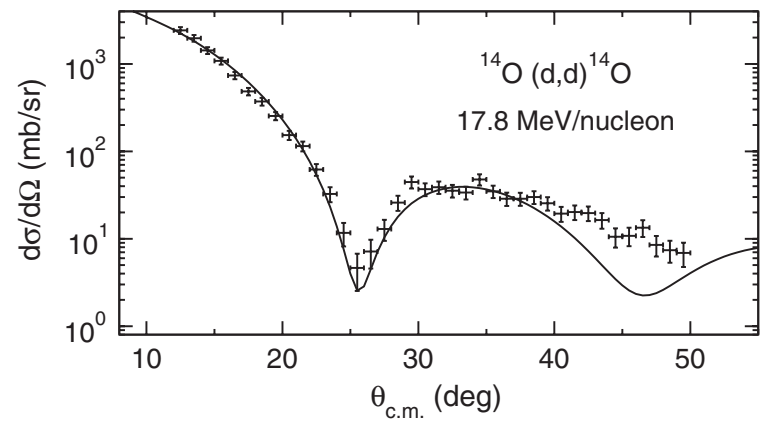

FIG. 1. Elastic scattering ${ }^{14} \mathrm{O}(d, d)$ cross section at $17.8 \mathrm{MeV} /$ nucleon and the result of the CRC calculation (solid line). channels, respectively, are plotted. To obtain optimum agreement with the measured distribution in Fig. 1, the folded entrance channel potential was renormalized by factors $\lambda_{V}=1.1$ and $\lambda_{W}=0.8$ for the real and imaginary parts, respectively. The overall agreement validates the choice of potentials and couplings used for the calculation of the transfer reactions.

The CRC calculations need a form factor to describe the transferred nucleon inside the light target and the heavy projectile. The form factors of Ref. [29] were used for the $\langle t \mid d+n\rangle$ and $\left\langle{ }^{3} \mathrm{He} \mid d+p\right\rangle$ overlaps. Two kinds of overlap function (OF) were examined: (i) a Woods-Saxon (WS) prescription with a constraint on the radius, and (ii) $a b$ initio microscopic OFs obtained from selfconsistent Green's function (SCGF) theory [30].

In the first approach, we used the wave function calculated in a WS potential with radius $r_{0} \times A^{1 / 3}$ and diffuseness $a_{0}=0.65 \mathrm{fm}$. Instead of the standard arbitrary value $r_{0}=1.25 \mathrm{fm}$, we chose, for ${ }^{16} \mathrm{O}$, the values $r_{0}=1.46$ and $1.31 \mathrm{fm}$ which reproduce the root mean square radii, $r_{\mathrm{rms}}=2.943$ and $2.719 \mathrm{fm}$, respectively, extracted from ${ }^{16} \mathrm{O}\left(e, e^{\prime} p\right){ }^{15} \mathrm{~N}_{\text {g.s. }}$ and ${ }^{15} \mathrm{~N}_{3 / 2-}$ [5]. The same $r_{0}$ value was adopted for the neutron wave function in ${ }^{15} \mathrm{O}_{\text {g.s. }}$. With no $\left(e, e^{\prime} p\right)$ data available for ${ }^{14} \mathrm{O}$ and ${ }^{18} \mathrm{O}$, we performed Hartree-Fock-Bogoliubov (HFB) calculations with Skyrme interactions to fix the radii of the $0 p_{3 / 2}$ and $0 p_{1 / 2}$ wave functions. This choice was validated by the
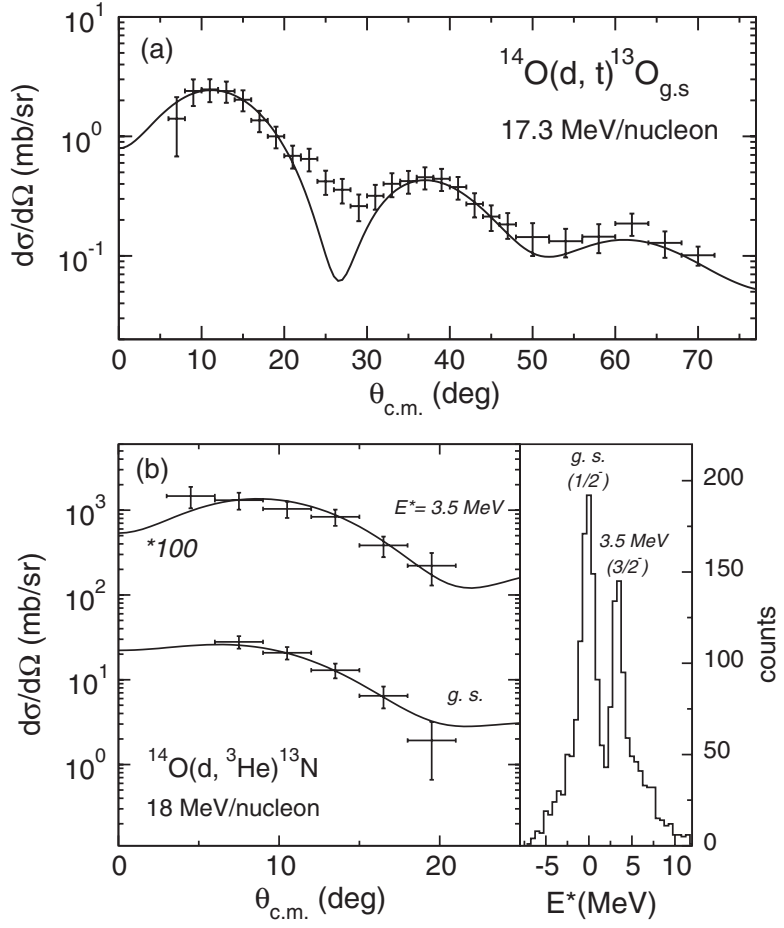

FIG. 2. The ${ }^{14} \mathrm{O}(d, t)$ and $\left(d,{ }^{3} \mathrm{He}\right)$ transfer reactions at 17.3 and $18 \mathrm{MeV} /$ nucleon (mid-target); (a),(b) (left) angular distributions; (b) (right) excitation energy spectrum. Solid lines are the CRC calculations assuming $\Delta l=1$. 


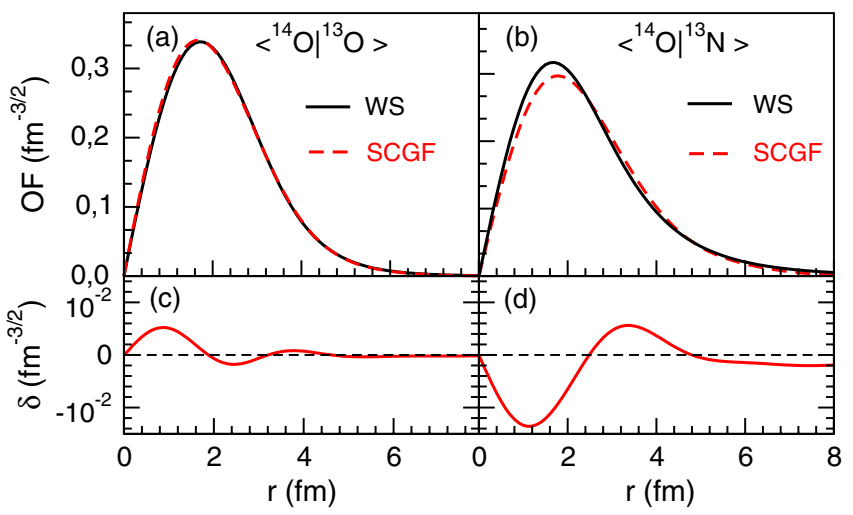

FIG. 3 (color online). Radial dependence of (a), (b) the OFs for WS and microscopic (SCGF) [30] form factors normalized to 1; (c), (d) the OF difference $\delta$ (SCGF - WS).

fair agreement obtained for the calculation of the ${ }^{16} \mathrm{O}$ rms radii performed with the SLy4 interaction [31] compared to the values deduced from ${ }^{16} \mathrm{O}\left(e, e^{\prime} p\right){ }^{15} \mathrm{~N}_{\mathrm{gs}}$ and ${ }^{15} \mathrm{~N}_{3 / 2-}$ analyses [5], both states with large SFs. We thus adopted the HFB radii calculated for the $0 p$ wave functions for ${ }^{14} \mathrm{O}$ and ${ }^{18} \mathrm{O}$ and deduced the corresponding values of $r_{0}$. The same calculation was done with other Skyrme interactions, always in fair agreement with the ${ }^{16} \mathrm{O}\left(e, e^{\prime} p\right)$ results, from which we deduced a variance for $r_{0}$.

The calculated angular distributions were normalized to the data by a factor $C^{2} S_{\text {exp }}$, which defines a so-called experimental SF. $C^{2} S_{\text {exp }}$ are mainly sensitive to the most forward angles, and so little sensitive to the details of the nuclear potentials. $C^{2} S_{\exp }$ strongly depend on radii with $\Delta S F / S F \approx 6 \Delta r_{\text {rms }} / r_{\text {rms }}$ in the ${ }^{14} \mathrm{O}(d, t)$ analysis.

We first reanalyzed published data for single nucleon pickup reactions at about the same incident energy in direct kinematics [19-21] on ${ }^{16} \mathrm{O}$ and ${ }^{18} \mathrm{O}$ targets. The angular distributions were well reproduced in all cases by CRC calculations. For ${ }^{16} \mathrm{O}\left(d,{ }^{3} \mathrm{He}\right)$ at 14 and $26 \mathrm{MeV} /$ nucleon, we obtained same $C^{2} S_{\text {exp }}$, which confirms the energy independence of the analysis. For the ${ }^{14} \mathrm{O}\left(d,{ }^{3} \mathrm{He}\right)$ and ${ }^{14} \mathrm{O}(d, t)$ transfers, the shape of the angular distributions is nicely reproduced (Fig. 2) by the CRC calculations assuming a $\Delta l=1$ transferred angular momentum, as expected from the transfer of a $0 p$ nucleon.

In the second approach, we employed ab initio SFs and OFs obtained from the single-particle Green's function in the third order algebraic diagrammatic construction method [ADC(3)] [14,32]. Calculations were based on chiral two-body next-to-next-to-next-to leading order (N3LO) [33] plus three-body next-to-next-to leading order (N2LO) [34] interactions evolved to a cutoff $\lambda=$ $1.88 \mathrm{fm}^{-1}$, as introduced in Ref. [35]. All microscopic OFs were further rescaled in coordinate space by the same factor (i.e., introducing only one phenomenological correction) to account for differences of predicted [30] and experimental rms radius of ${ }^{16} \mathrm{O}$. The OFs corresponding to the removal of main peaks at large and small nucleon separation energies are shown in Figs. 3(a) and 3(b), respectively, and compared to the Wood-Saxon prescription. We note very little radial difference in the removal of the strongly bound neutron in ${ }^{14} \mathrm{O}$.

We give in Table I the normalizations $C^{2} S_{\text {exp }}$ for the two kinds of OFs. From theoretical SFs inputs, either microscopic ab initio SFs [30] or shell-model SFs, we obtain a theoretical value $\sigma_{\mathrm{th}}(\theta)$ and the reduction factor $R_{s}=$ $\sigma_{\exp }(\theta) / \sigma_{\text {th }}(\theta)$. For shell-model SFs, we performed two calculations with different valence space and interaction: (i) in the $0 p+2 \hbar \omega$ valence space with Oxbash [36] and the WBT interaction [37] shown in Table I (here the active orbitals are $0 p_{3 / 2}$ and $0 p_{1 / 2}$ and only $2 p 2 h$ excitations toward the $s d$ orbitals are allowed), and (ii) in the $0 p 1 s 0 d$ valence space with Nushellx [38] and a new interaction [39]. With the WBT interaction, we find good agreement for the energies of the listed states, while with the new interaction the energies of excited states in ${ }^{13} \mathrm{~N}$ and ${ }^{15} \mathrm{~N}$ disagree by several MeV. Finally, we show the reduction factor $R_{s}$, also plotted in Figs. 4(a) and 4(b), for WS and microscopic OFs, respectively. In the total uncertainty, we set apart in a box the uncertainties originating from the analysis: (i) imperfect knowledge of entrance and exit potentials, and (ii) the variance in the calculation of rms

TABLE I. The normalization $C^{2} S_{\text {exp }}$ for two OFs, phenomenological (WS) and microscopic (SCGF) [30]. For the WS OF, the $r_{0}$ values were chosen to reproduce $R_{\mathrm{rms}}^{\mathrm{HFB}}$, except for ${ }^{16} \mathrm{O}$ for which $R_{\mathrm{rms}}$ was taken from $\left(e, e^{\prime} p\right)$ data (see text). The SFs $C^{2} S_{\mathrm{th}}$ are obtained from shell-model calculations with the WBT interaction. In the second part, the analysis was performed with microscopic OFs and SFs. The two errors for $C^{2} S_{\text {exp }}$ and $R_{s}$ are the experimental and analysis errors.

\begin{tabular}{|c|c|c|c|c|c|c|c|c|c|c|}
\hline Reaction & $E^{*}(\mathrm{MeV})$ & $J^{\pi}$ & $\begin{array}{l}R_{\mathrm{rms}}^{\mathrm{HFB}} \\
(\mathrm{fm})\end{array}$ & $\begin{array}{c}r_{0} \\
(\mathrm{fm})\end{array}$ & $\begin{array}{l}C^{2} S_{\exp } \\
(\mathrm{WS})\end{array}$ & $\begin{array}{c}C^{2} S_{\mathrm{th}} \\
0 p+2 \hbar \omega\end{array}$ & $\begin{array}{c}R_{S} \\
(\mathrm{WS})\end{array}$ & $\begin{array}{c}C^{2} S_{\exp } \\
(\mathrm{SCGF})\end{array}$ & $\begin{array}{c}C^{2} S_{\mathrm{th}} \\
(\mathrm{SCGF})\end{array}$ & $\begin{array}{c}R_{s} \\
(\mathrm{SCGF})\end{array}$ \\
\hline${ }^{14} \mathrm{O}(d, t){ }^{13} \mathrm{O}$ & 0.00 & $3 / 2^{-}$ & 2.69 & 1.40 & $1.69(17)(20)$ & 3.15 & $0.54(5)(6)$ & $1.89(19)(22)$ & 3.17 & $0.60(6)(7)$ \\
\hline \multirow[t]{2}{*}{${ }^{14} \mathrm{O}\left(d,{ }^{3} \mathrm{He}\right){ }^{13} \mathrm{~N}$} & 0.00 & $1 / 2^{-}$ & 3.03 & 1.23 & $1.14(16)(15)$ & 1.55 & $0.73(10)(10)$ & $1.58(22)(2)$ & 1.58 & $1.00(14)(1)$ \\
\hline & 3.50 & $3 / 2^{-}$ & 2.77 & 1.12 & $0.94(19)(7)$ & 1.90 & $0.49(10)(4)$ & $1.00(20)(1)$ & 1.90 & $0.53(10)(1)$ \\
\hline${ }^{16} \mathrm{O}(d, t){ }^{15} \mathrm{O}$ & 0.00 & $1 / 2^{-}$ & 2.91 & 1.46 & $0.91(9)(8)$ & 1.54 & $0.59(6)(5)$ & $0.96(10)(7)$ & 1.73 & $0.55(6)(4)$ \\
\hline \multirow[t]{2}{*}{${ }^{16} \mathrm{O}\left(d,{ }^{3} \mathrm{He}\right){ }^{15} \mathrm{~N}[19,20]$} & 0.00 & $1 / 2^{-}$ & 2.95 & 1.46 & $0.93(9)(9)$ & 1.54 & $0.60(6)(6)$ & $1.25(12)(5)$ & 1.74 & $0.72(7)(3)$ \\
\hline & 6.32 & $3 / 2^{-}$ & 2.80 & 1.31 & $1.83(18)(24)$ & 3.07 & $0.60(6)(8)$ & $2.24(22)(10)$ & 3.45 & $0.65(6)(3)$ \\
\hline${ }^{18} \mathrm{O}\left(d,{ }^{3} \mathrm{He}\right){ }^{17} \mathrm{~N}[21]$ & 0.00 & $1 / 2^{-}$ & 2.91 & 1.46 & $0.92(9)(12)$ & 1.58 & $0.58(6)(10)$ & & & \\
\hline
\end{tabular}




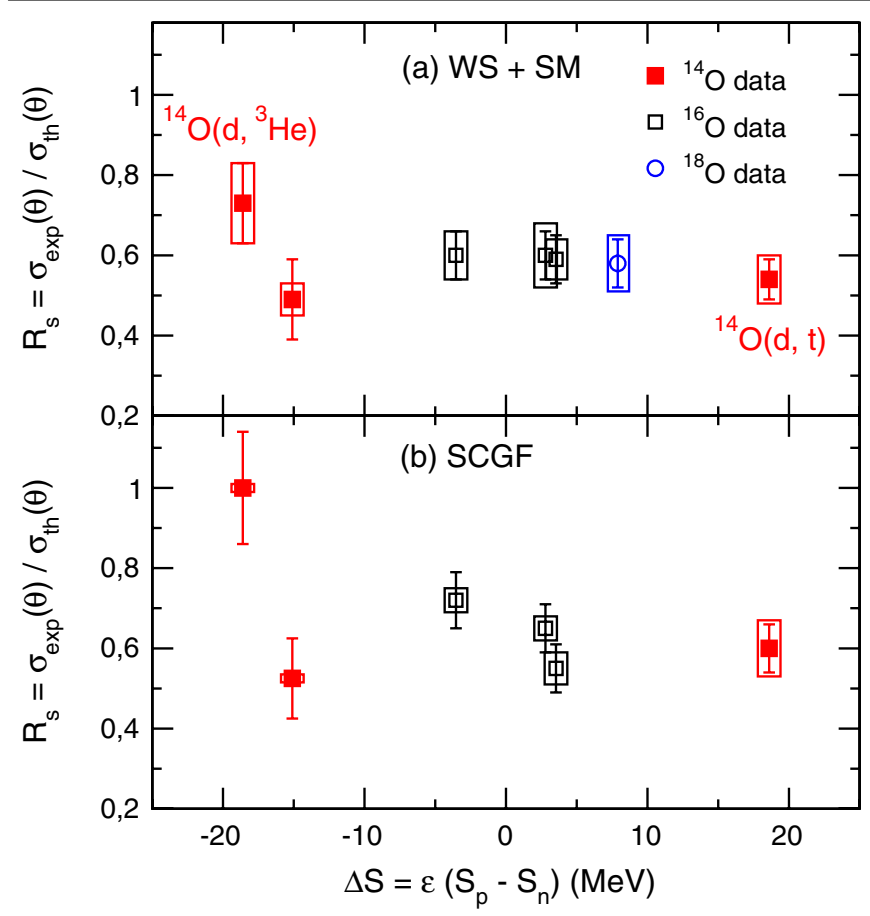

FIG. 4 (color online). Reduction factors $R_{s}$ obtained with (a) a WS OF and the SLy4 interaction [31], averaged over four entrance and two exit potentials, and compared to shell-model calculations performed with the WBT interaction [37] in the $0 p+2 \hbar \omega$ valence space; (b) a microscopic (SCGF) form factor [30]. The detail of error bars is given in text.

radii (and consequently of $r_{0}$ ) due to different Skyrme interactions, provided the rms radii of ${ }^{15} \mathrm{~N}$ extracted from $\left(e, e^{\prime} p\right)$ [5] are reproduced. All the other experimental uncertainties are accounted for by the error bars displayed on Fig. 4. A rather flat trend is found without the need for the large asymmetry dependence suggested by intermediate energy knockout data analyzed with the eikonal formalism [10]. For a quantitative evaluation, we fitted the reduction factor with a linear dependence $R_{s}=$ $\alpha \times \Delta S+\beta$. We obtained mean values for $\alpha$ and $\beta$ with associated errors from a minimization over the 48 data sets, considering (i) eight combinations of optical potentials for the entrance and exit channels, (ii) three Skyrme interactions to calculate the rms radii, and (iii) the two abovementioned shell-model calculations.

For the WS OF, the reduction factor $R_{s}=0.538(28)(18)$ (for $\Delta S=0$ nuclei) is in agreement with Ref. [9] and the slope parameter $\alpha=0.0004(24)(12) \mathrm{MeV}^{-1}$, therefore consistent with zero. The first standard error obtained over one data set depends on the experimental uncertainties; the second one comes from the distribution over the 48 data sets. Within the error bars, the data do not contradict the weak dependence found by ab initio calculations, with $\alpha^{\prime}=-0.0039 \mathrm{MeV}^{-1}$ between the two ${ }^{14} \mathrm{O}$ points in Ref. [7], although the calculated $\Delta S$ is much reduced compared to the experimental value.
Despite different OFs and SFs, the analysis performed with the $a b$ initio OF [30] provides very similar results with $R_{s}(\Delta S=0)=0.636(34)(42)$ and $\alpha=$ $-0.0042(28)(36) \mathrm{MeV}^{-1}$, with calculated $\Delta S=17.6 \mathrm{MeV}$ [Fig. 4(b)].

In summary, we measured exclusive differential cross sections at $18 \mathrm{MeV} /$ nucleon for the ${ }^{14} \mathrm{O}(d, t){ }^{13} \mathrm{O}$ and ${ }^{14} \mathrm{O}\left(d,{ }^{3} \mathrm{He}\right){ }^{13} \mathrm{~N}$ transfer reactions and elastic scattering. WS OFs with a constraint on HF radii and microscopic OFs (obtained from SCFG theory) have been compared for the first time for symmetric and very asymmetric nuclei and gave similar results. We extracted the reduction factors $R_{s}$ over a high asymmetry range, $\Delta S= \pm 18.5 \mathrm{MeV}$, for oxygen isotopes. From the good agreement between the CRC calculations and the set of transfer data highlighted in our work, the asymmetry dependence is found to be nonexistent (or weak), within the error bars. This result is in agreement with $a b$ initio Green's function and coupledcluster calculations [7,14], but contradicts the trend observed in nucleon knockout data obtained at incident energies below $100 \mathrm{MeV} /$ nucleon and analyzed with the sudden-eikonal formalism. The disagreement of the two systematic trends from knockout and transfer calls for a better description of so-called direct reaction mechanisms in order that a consistent picture of nuclear structure emerges from measurements at different incident energies.

The authors thank N. T. Timofeyuk and N. Alamanos for enlightening discussions and P. Navrátil for providing evolved two- and three-body interactions relevant to this study. This work was supported by LIA COPIGAL and POLONIUM PHC under Grant No. 22470XA. Theoretical work was supported by the UK's STFC Grant No. ST/ J000051/1.

[1] W. H. Dickhoff and C. Barbieri, Prog. Part. Nucl. Phys. 52, 377 (2004).

[2] S. Boffi et al., Electromagnetic Response of Atomic Nuclei, Oxford Studies in Nuclear Physics Vol. 20 (Clarendon Press, Oxford, 1996).

[3] M. Bernheim et al., Nucl. Phys. A375, 381 (1982).

[4] L. Lapikas, Nucl. Phys. A553, 297 (1993).

[5] M. Leuschner et al., Phys. Rev. C 49, 955 (1994).

[6] C. Barbieri, Phys. Rev. Lett. 103, 202502 (2009).

[7] Ø. Jensen, G. Hagen, M. Hjorth-Jensen, B. A. Brown, and A. Gade, Phys. Rev. Lett. 107, 032501 (2011).

[8] G. J. Kramer, H.P. Blok, and L. Lapikás, Nucl. Phys. A679, 267 (2001).

[9] J. P. Schiffer et al., Phys. Rev. Lett. 108, 022501 (2012).

[10] A. Gade et al., Phys. Rev. C 77, 044306 (2008).

[11] C. Louchart, A. Obertelli, A. Boudard, and F. Flavigny, Phys. Rev. C 83, 011601(R) (2011).

[12] F. Flavigny, A. Obertelli, A. Bonaccorso, G. F. Grinyer, C. Louchart, L. Nalpas, and A. Signoracci, Phys. Rev. Lett. 108, 252501 (2012).

[13] J. Lee et al., Phys. Rev. C 83, 014606 (2011). 
[14] C. Barbieri and W. H. Dickhoff, Int. J. Mod. Phys. A 24, 2060 (2009).

[15] E. C. Pollacco et al., Eur. Phys. J. A 25, 287 (2005).

[16] S. Pullanhiotan, M. Rejmund, A. Navin, W. Mittig, and S. Bhattacharyya, Nucl. Instrum. Methods Phys. Res., Sect. A 593, 343 (2008).

[17] S. Ottini-Hustache et al., Nucl. Instrum. Methods Phys. Res., Sect. A 431, 476 (1999).

[18] F. Ajzenberg-Selove, Nucl. Phys. A523, 1 (1991).

[19] V. Bechtold, L. Friedrich, P. Doll, K. T. Knöpfle, G. Mairle, and G. J. Wagner, Phys. Lett. B 72, 169 (1977).

[20] M. Gaillard, R. Bouché, L. Feuvrais, P. Gaillard, A. Guichard, M. Gusakow, J. L. Leonhardt, and J.-R. Pizzi, Nucl. Phys. A119, 161 (1968).

[21] D. Hartwig, G. Th. Kaschl, G. Mairle, and G. J. Wagner, Z. Phys. 246, 418 (1971).

[22] I. J. Thompson, Comput. Phys. Rep. 7, 167 (1988).

[23] C. M. Perey and F. G. Perey, At. Data Nucl. Data Tables 17, 1 (1976).

[24] D. Y. Pang, P. Roussel-Chomaz, H. Savajols, R. L. Varner, and R. Wolski, Phys. Rev. C 79, 024615 (2009).

[25] A. J. Koning and J. P. Delaroche, Nucl. Phys. A713, 231 (2003).
[26] R. L. Varner, W. J. Thompson, T. L. McAbee, E. J. Ludwig, and T. B. Clegg, Phys. Rep. 201, 57 (1991).

[27] F. D. Becchetti and G. W. Greenlees, Phys. Rev. 182, 1190 (1969).

[28] B. A. Watson, P. P. Singh, and R. E. Segel, Phys. Rev. 182, 977 (1969).

[29] A. M. Eiró and I. J. Thompson, Phys. Rev. C 59, 2670 (1999).

[30] C. Barbieri et al., arXiv:1211.3315.

[31] E. Chabanat, P. Bonche, P. Haensel, J. Meyer, and R. Schaeffer, Nucl. Phys. A635, 231 (1998).

[32] C. Barbieri, D. Van Neck, and W. H. Dickhoff, Phys. Rev. A 76, 052503 (2007).

[33] D. R. Entem and R. Machleidt, Phys. Rev. C 68, 041001 (R) (2003).

[34] P. Navrátil, Few-Body Syst. 41, 117 (2007).

[35] R. Roth, S. Binder, K. Vobig, A. Calci, J. Langhammer, and P. Navrátil, Phys. Rev. Lett. 109, 052501 (2012).

[36] B. A. Brown et al., MSU-NSCL Report No. 524, 1985.

[37] E. K. Warburton and B. A. Brown, Phys. Rev. C 46, 923 (1992).

[38] B. A. Brown, http://www.nscl.msu.edu/ brown.

[39] Y. Utsuno and S. Chiba, Phys. Rev. C 83, 021301(R) (2011). 